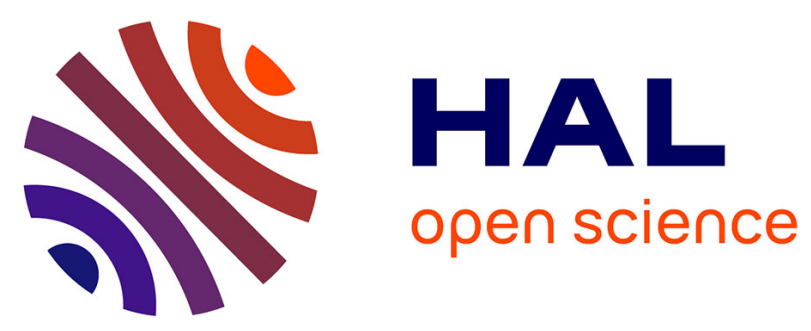

\title{
Epithelial to mesenchymal transition-The roles of cell morphology, labile adhesion and junctional coupling.
} Tariq Abdulla, Jean-Marc Schleich, Ron Summers

\section{To cite this version:}

Tariq Abdulla, Jean-Marc Schleich, Ron Summers. Epithelial to mesenchymal transition-The roles of cell morphology, labile adhesion and junctional coupling.. Computer Methods and Programs in Biomedicine, 2013, 111 (2), pp.435-446. 10.1016/j.cmpb.2013.05.018 . inserm-00838163

\section{HAL Id: inserm-00838163 https://www.hal.inserm.fr/inserm-00838163}

Submitted on 24 Jun 2013

HAL is a multi-disciplinary open access archive for the deposit and dissemination of scientific research documents, whether they are published or not. The documents may come from teaching and research institutions in France or abroad, or from public or private research centers.
L'archive ouverte pluridisciplinaire HAL, est destinée au dépôt et à la diffusion de documents scientifiques de niveau recherche, publiés ou non, émanant des établissements d'enseignement et de recherche français ou étrangers, des laboratoires publics ou privés. 


\section{Epithelial to Mesenchymal Transition - the Roles of Cell Morphology, Labile Adhesion and Junctional Coupling}

Tariq Abdulla (1), Luis Luna-Zurita (2), José Luis de la Pompa (2), Jean-Marc Schleich (3) and Ron Summers (1)

(1) School of Electronic, Electrical and Systems Engineering, Loughborough University, Loughborough, UK

(2) Program of Cardiovascular Developmental Biology, Department of Cardiovascular Development and Repair, Centro Nacional de Investigaciones Cardiovasculares (CNIC), Melchor Fernández Almagro 3, 28029 Madrid, SPAIN

(3) Université de Rennes 1, LTSI, 35000 Rennes, France

\section{Abstract}

Epithelial to mesenchymal transition (EMT) is a fundamental process during development and disease, including development of the heart valves and tumour metastases. An extended cellular Potts model was implemented to represent the behaviour emerging from autonomous cell morphology, labile adhesion, junctional coupling and cell motility. Computer simulations normally focus on these functional changes independently whereas this model facilitates exploration of the interplay between cell shape changes, adhesion and migration. The simulation model is fitted to an in vitro model of endocardial EMT, and agrees with the finding that Notch signalling increases cell-matrix adhesion in addition to modulating cell-cell adhesion.

\section{Background}

\subsection{Biological Background}

Epithelial to mesenchymal transition (EMT) is the process during which cells lose epithelial characteristics, and acquire mesenchymal features. This includes changes in cell shape, migratory characteristics and changes in expression of cell adhesion molecules. EMT is fundamental to a multitude of embryonic processes in vertebrate development, including gastrulation, somitogenesis, neural crest cell migration and the development of heart valves (see Figure 1). EMT-like processes take place during adult epithelial wound healing and in cancer progression [1]. These EMT and EMT-like processes are diverse and are regulated by a wide range of signalling pathways in different tissues and organs, with extensive crosstalk between pathways. However they have as a common endpoint in: the downregulation of E-Cadherin and thus loss of epithelial adhesion; the up-regulation of mesenchymal genes such as vimentin and fibronectin; and as a consequence of these two processes a gain of cell to matrix adhesion and migratory or invasive behaviour [2]. Furthermore, the rearrangement of proteins, e.g. the relocation of actin and E-cadherin from adherens junctions to the cytoskeleton, enables the formation of stress fibres that give cells greater strength, flexibility and migration capacity [3]. The secretion of matrix metalloproteinases, which degrade extracellular matrix proteins (including those in the basement membrane), is also key to transformation and invasion in many types of EMT [4]. It is important to make 
a distinction between migration and invasion. For the purpose of this study, invasion involves the degradation of extracellular matrix proteins, whereas migration involves cell movement without degradation of matrix proteins. This definition follows from in vitro experiments in which '2D transformation' (migration) is measured separately from '3D transformation' (invasion). Notch activation and/or treatment with TGF $\beta$ protein induced only migration on the surface of the collagen gel, whereas treatment with BMP2 enabled the cells to degrade the collagen and invade into it [5]. In this model only the changes that take place during 2D migration are measured.

a)

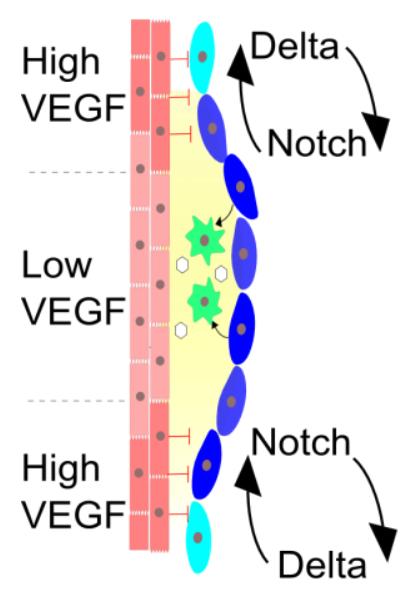

b)

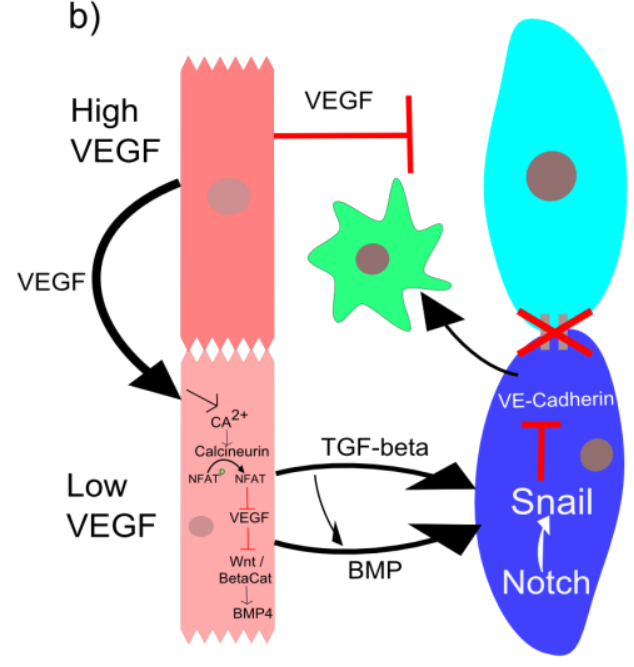

c)

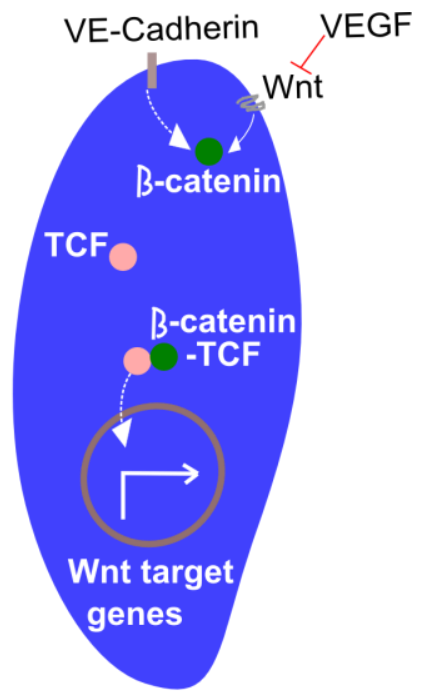

d)

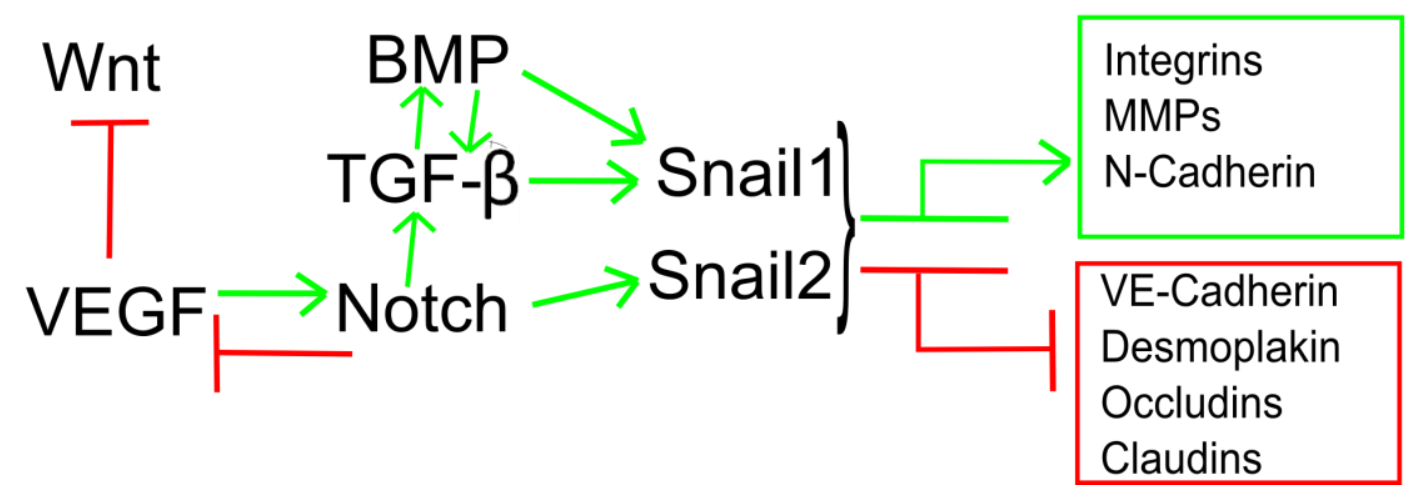

Figure 1 a) During heart development, a field of cells with the potential to undergo EMT is specified by endocardial Notch signalling, and modulated VEGF secretion from the myocardium. b) The lower VEGF signalling causes BMP and TGF- $\beta$ secretion from the myocardium. This cooperates with Notch signalling to disassemble VE-cadherin adherens junctions, reducing endocardial cohesion. $c$ ) Disassembly of adherens junctions leads to $\beta$-catenin no longer being sequestered in the cytoplasm. It thus becomes available to be used in the Wnt signalling pathway, and translocates to the nucleus. VEGF inhibition of Wnt is one means by which VEGF modulates EMT. d) Snail genes up-regulate mesenchymal markers while down-regulating epithelial markers, and are downstream of Notch, TGF- $\beta$ and BMP signalling. These pathways exhibit crosstalk, indicative of their concerted activation in cardiac EMT.

In endocardial cells the Notch1 protein is known to induce EMT by up-regulating Snail proteins, which in turn acts to down-regulate VE-Cadherin [5]. Cell doublet experiments have been used to measure the strength of cell adhesion as a function of contact time. In these experiments, two E-cadherin expressing cells are held in contact and the force required to separate them measured after given durations [6]. 
The adhesion force required to separate two E-cadherin expressing cells is initially of the order of a few nano Newtons $(\mathrm{nN})$. However, this adhesion strength increases rapidly when neighbouring cells remain in contact between 30 seconds and 30 minutes, followed by a slower increase when contact duration is between 30 minutes and an hour, reaching a peak force over $200 \mathrm{nN}$ [6]. The initial E-cadherin mediated contact adhesion does not require connection to the actin cytoskeleton. The stronger junctional adhesion forms over a longer period of contact, by connection to the actin cytoskeleton between the two cells.

There are a number of examples of crosstalk between the protein signalling pathways which indicate that loss of epithelial adhesion cannot be decoupled from an increase in matrix adhesion. For example, the disassembly of cadherin-mediated junctions leads to the release of $\beta$-catenin which is then transferred to the nucleus. The $\beta$-catenin then induces expression of Wnt target genes. The effects of Wnt/ $\beta$-catenin signalling include further down-regulation of E-cadherin, and up-regulation of integrins and $\mathrm{N}$-cadherin, which favour matrix adhesion and cell motility [7]. There is great complexity in this regulation due to the crosstalk between the Notch and TGF $\beta$ pathways, which target each of the Snail genes to different extents [8]. Additionally, the Hey genes (also Notch targets) repress the BMP pathway, and the BMP pathway exhibits further crosstalk with TGF [5]. While in vitro and in vivo experiments investigate the effects of individual proteins on EMT, independent quantitative control of biological mechanisms is difficult. Computational models complement experimental work by allowing for the exploration of different combinations of physical parameters.

\subsection{Technical Background}

There are three main types of agent-based modelling that can be used for representing cell behaviour: cell centre, cell vertex and cellular Potts models (see Figure 2). In cell centre models, the centres of cells are represented as points in space. Forces are defined as acting between these centres, such as damped spring forces and connectivity between cells can then be represented by Voronoi tessellation [9]. In cell vertex models, cells are represented as adjacent polygons with vertices that move as a result of the balance of forces between cells. The dynamics of each cell are represented by the movement of its vertices. This movement can either be determined by explicit forces on each vertex or by a free energy function. It is thus possible to provide direct model representation of mechanical forces such as plasticity, elasticity and viscoelasticity of cells, as well as incorporating differential adhesion terms [10]. Finally in cellular Potts models, cells are represented as multiple sites on a lattice. Cell dynamics are represented by pixel copy attempts between adjacent lattice sites belonging to different cells. These are accepted with a probability that depends on a free energy function, allowing differential adhesion and properties of cell morphology and migration to be modelled. Of the three methods, cellular Potts modelling allows the most direct representation of cell shape [11]. 
a) Cell
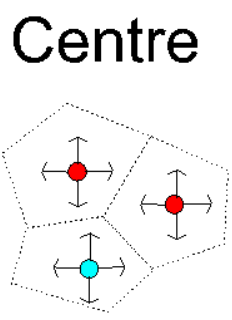

b) Cell Vertex

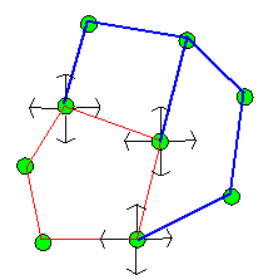

c) Cellular Potts

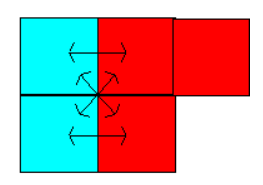

Figure 2 a) Cell centre, b) Cell vertex and c) Cellular Potts model representations.

Various types of EMT have been modelled using these different agent- based simulation methods. Wound healing EMT has been modelled using a cell centre model [12], as has cancer metastasis EMT [13]. These models both incorporate subcellular reaction models, and represent cell adhesion as a breakable spring force between cell centres. Endocardial EMT has been modelled using the cellular Potts method [14]. Although the latter study did not investigate the role of cell morphology during EMT, the cellular Potts method is suitable for doing this, as it includes an explicit representation of cell shape. Furthermore, by incorporating a plastic coupling term within the cellular Potts model, it is possible to design a framework for investigating the interactions of cell morphology, labile adhesion and junctional coupling. This methodology is explained further in section 2.1 below.

\section{Methods}

\subsection{Cellular Potts Model}

Cellular Potts models (CPMs) are lattice-based simulations with cells occupying multiple sites on the lattice. The main advantage of this schema is that it allows cell shape to be explicitly represented. Thus the simulations have the potential to describe processes in which control of cell shape plays an important role. According to the Differential Adhesion Hypothesis (DAH), morphogenetic changes are driven by cell displacements that lead to the lowest energy configurations, and thus the largest number of strong adhesive bonds. This can be simulated with CPMs using a Metropolis Monte Carlo algorithm. A Hamiltonian energy, $H$, is defined for the system. During each step in the simulation, a random copy attempt is made for each lattice site at a cell surface. For each copy attempt, the resulting change in energy, $\Delta H$, is calculated, and the copy attempt is accepted with a probability $\min \left(1, e^{\Delta H / T}\right)$; where $T$ is used as an intrinsic measure of cell motility. Motile cells in a CPM will tend to move so as to reduce $H$, reducing the entropy of the system. Typically, the Hamiltonian equation includes terms for type dependent surface energies between each pair of different cell types. This represents their level of adhesion. Higher surface energy represents a lower level of labile adhesion. CPM can be extended to include terms for anything that can be calculated from the cell attributes. For example, a type dependent target volume or target surface area can be included, with values for the propensity of a cell to reach the target. One of the simplest cellular Potts simulations represents a cell sorting experiment, where an initial mixed population of two or more cell types become sorted (see Figure 3 ). The cells with 
higher preferential cohesion move to the centre of the cluster, while those with lower cohesion move to outer layers.
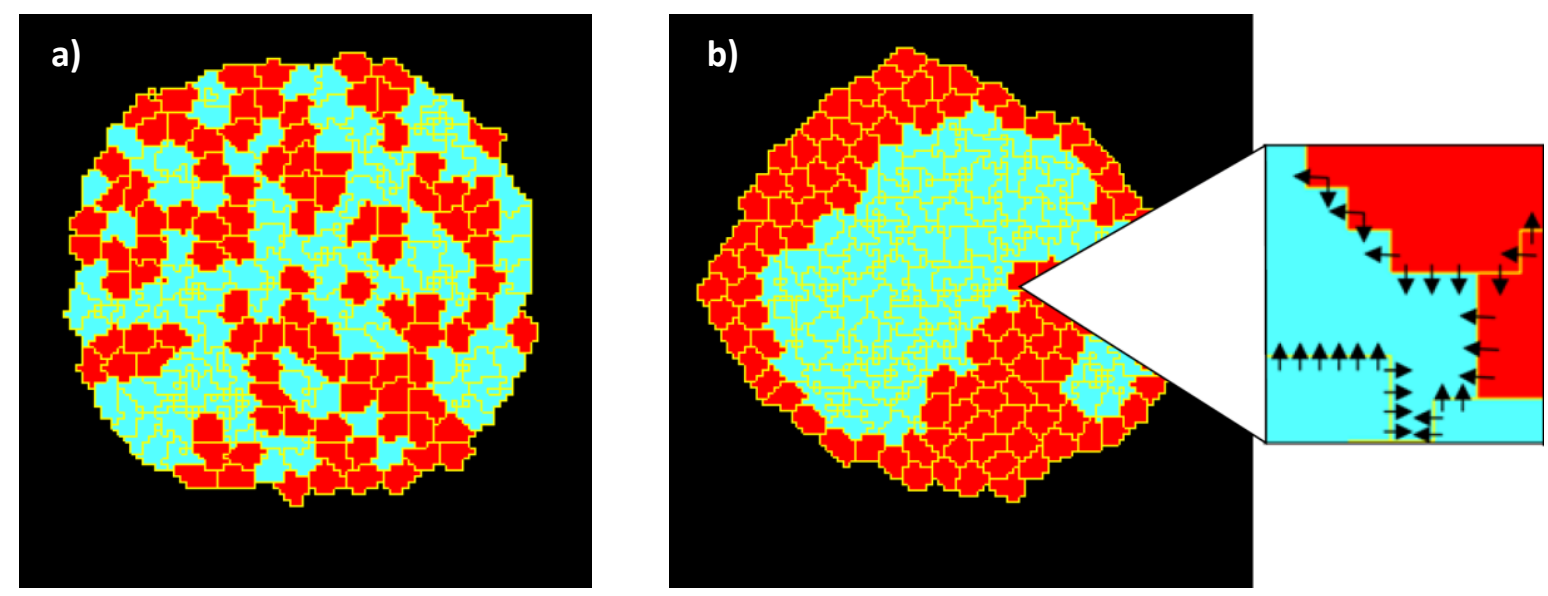

Figure 3 Cells interact at their surfaces (adjacent lattice sites) in cellular Potts models. a) At the start of the simulation, cell types are mixed. b) After $\mathbf{8 0 0 0}$ Monte Carlo Steps (MCS), the cells are sorted, with the less cohesive type forming an outer layer.

CPMs are able to simulate cell behaviour by representing any mechanism where cell rearrangement is determined principally by differences in adhesion. As the focus of CPM is cell reorganisation, they have been used mainly for modelling developmental mechanisms. Compucell3D [15] is the most widely used modelling environment for implementation of CPMs; it is an open source resource and extensible, which enable the sharing of results.

Compucell3D simulations of individual cells were used to simulate the changes in cell shape. By using an individual cell, multiple parameters on the interplay of cell morphology and migration could be explored. To give control over cell shape, terms for volume and surface constraints are added to the contact term in the Hamiltonian equation. An elongation term is used for simulations that investigate the effect of elongation, with a connectivity penalty to ensure that elongated and fibroblastic cells do not fragment. Finally, part of the parameter space is implemented in 2D multicellular simulations of generic in vitro epithelial tissue. A term for plastic coupling is added to the multicellular simulations, this allows for investigation of the roles of labile adhesion (contact energy) and junctional coupling (plastic energy) in determining epithelial morphology. Thus in these simulations, the Hamiltonian energy, $\mathrm{H}$, is given by the following equation:

$$
H=E_{\text {Contact }}+E_{\text {Plastic }}+E_{\text {Surface }}+E_{\text {Volume }}+E_{\text {Length }}+E_{\text {Connectivity }}
$$

Labile adhesion is represented by a negative contact energy, $J$, between cells. The more negative this number is, the greater the level of labile adhesion between cells:

$$
E_{\text {Contact }}=\sum_{\mathrm{i}, \mathrm{j}-\text { pixel ni ghbours }} \mathrm{J}\left[\tau_{\sigma(\mathrm{i})}, \tau_{\sigma(\mathrm{j})}\right]\left[1-\delta_{\sigma(\mathrm{i}), \sigma(\mathrm{j})}\right]
$$


Where $i$ and $j$ label two neighbouring lattice sites, $\sigma$ denotes cell identifiers, $\tau$ denotes cell types, and the Kronecker delta is $\delta_{x y y}=\{1, x=y ; 0, x \neq y\}$.

Plastic coupling is represented by a breakable spring force between cell centres, and this term is added to the Hamiltonian equation governing the simulations, giving:

$E_{\text {Plastic }}=\sum_{i, j-\text { cell neighbours }} \lambda_{i j}\left(p_{i j}-P_{i j}\right)^{2}$

where $p_{i j}$ is the distance between the centre of masses of cells $i$ and $j ; P_{i j}$ is the target distance corresponding to $p_{i j}$, and $\lambda_{i j}$ is a constraint representing the strength of the plastic coupling.

Additionally, a maximum distance is set, which determines the distance between the centre of mass of neighbouring cells when the link between them breaks, and also the distance at which links can be established between unconnected cells.

The volume and surface constraint terms are given by:

$$
\begin{aligned}
& E_{\text {Surface }}=\sum_{\text {cells }} \lambda_{\text {Surface }}(s-S)^{2} \\
& E_{\text {Volume }}=\sum_{\text {cells }} \lambda_{\text {volume }}(v-V)^{2} \\
& E_{\text {Elogation }}=\sum_{\text {cells }} \lambda_{\text {Length }}(l-L)^{2}
\end{aligned}
$$

Where $\lambda_{\text {Surface }} \lambda_{\text {Volume }}$ and $\lambda_{\text {Length }}$ denote the strength of volume, surface and length constraints; $v$, $s$ and $/$ denote the current volume, surface and major axis length; and $V, S$ and $L$ denote the target volume, surface and length.

It would be possible to simulate an increase in cell motility in a number of different ways. For example, one could apply a randomly directed force to a cell, which fluctuates over time. However, this approach would introduce separate effects on cell shape, due to fluctuating cell polarisation. Whether Notch activated endocardial cells exhibit some form of directed movement and the specific dynamics of their movement (e.g. persistency) are more complicated questions which merit further investigation. In the simulations presented in this paper, an increase in cell motility is investigated by increasing its intrinsic measure, the $T$ parameter. This is the simplest and most direct way of increasing motility in cellular Potts simulations, as a greater proportion of the stochastic pixel copy attempts are accepted. As increases in the $T$ parameter or cell elongation might lead to fragmentation, which would be unrealistic, a connectivity constraint is used. The connectivity constraint is a large energy penalty $\left(10^{7}\right)$ for a pixel copy that would lead to cell fragmentation. 
All simulations presented in this paper are 2D rather than 3D. This simplification allows the comparison of simulation results to cell shape metrics acquired from 2D imaging data. The simplification is based on the assumption that cells are evenly $1 \mu \mathrm{m}$ in thickness; and lie approximately flat on the surface of the collagen gel. This is a reasonable assumption as it is typical of the morphology of endocardial cells. In this investigation the focus is on cells that migrate only on the surface of the gel, without the capability to invade it. This provides the justification for using 2D models.

\subsection{Fitting Space and Time Scale of Simulations}

The length scale was set to $1 \mu \mathrm{m}$ per pixel, and model cells given a width of $15 \mu \mathrm{m}$, based on the dimensions of cultured murine endocardial cells [5]. This choice reflects a prospective goal to include subcellular reaction kinetics; and thereby develop a multi-scale model of the integrated roles of Notch and TGF- $\beta$ signalling in EMT. Experimentally, in vitro epithelial cells such as MCF-7 cells move at a rate of about $0.28 \mu \mathrm{m} / \mathrm{min}$, whereas mesenchymal cells move at about $0.4 \mu \mathrm{m} / \mathrm{min}$ [16]. For typical parameter settings, simulated epithelial cells move at about 0.01 pixels/MCS (e.g. $0.01 \mu \mathrm{m} / \mathrm{MCS}$ ). Equating the experimental and simulated cell speed implies $0.28 \mathrm{MCS}=0.01 \mathrm{~min}$, or $1 \mathrm{MCS}=0.036 \mathrm{~min}$ (about 2 seconds). Each multicellular simulation was run for $5 \times 10^{4} \mathrm{MCS}$, which equates to 27.8 hours. Cellular migration and morphology was found to be quite stable after this time.

\subsection{Cell Morphology Metrics}

There are a few measures that can be used for quantifying the shape of cells. The main criteria for selecting suitable metrics is that they should enable a comparison to be made between the in vitro results [5] and the simulation results, that they should describe shape property relevant to the changes which take place during EMT, and preferably that they should be widely used in other experimental work, to allow for direct comparisons, making the results re-usable. Based on these criteria, the two metrics selected were aspect ratio (length/width) and circularity $\left(4 \pi^{*}\right.$ area/perimeter $\left.{ }^{2}\right)$. Aspect ratio indicates the elongation of a cell. Circularity gives a number between 0 and 1 , where a score of 1 indicates a perfect circle, and smaller values indicating a less rounded shape. These metrics can either be calculated during a simulation run, from basic cell attributes, or calculated from the confocal images of endocardial explants using ImageJ. This allows a direct comparison to be made between simulated representations and in vitro cells.

\subsection{Cell Migration Metrics}

There are a plethora of metrics that are used to quantify cell migration. For example, in vitro assays sometimes report the average speed of cells [17], and sometimes the average velocity [16]. Alternatively, the average displacement of cells over time can be measured [18]. When it comes to tissue simulations there are even more possibilities, because the migration of an individual cell can also be quantified by its relation to other cells. For example, it is possible to measure the average contact area between cells, the average number of cell neighbours or the ratio of cells that remain adhered to other cells [19]. Some investigations define their own metrics, for example, one study defined a 2D and 3D transformation index as the ratio of cells able to migrate on the surface, or invade the collagen gel [5]. It is even possible 
to analyse cell centre coordinates (provided by DAPI stained nuclei) by constructing a cell graph. Analysis of this graph provides metrics such as the mean and standard deviation of edge lengths, and a clustering coefficient [20]. It is possible to implement all of these metrics in simulations. Indeed it is more straightforward to measure and analyse this information from simulation runs than from in vitro experiments. However, in order to provide validation, the average speed, velocity and displacement remain the most suitable metrics to determine cell migration, as they are the most widely used in experimental work. In the case of the simulations presented in this paper, displacement is defined as the Euclidian distance between the current position of the centre of mass coordinates of a cell and its starting value. Velocity is defined as displacement, divided by the total time elapsed in a simulation. Speed is defined as displacement over the previous 100 simulation steps (equivalent to 3.6 minutes).

\subsection{Image Processing}

Further analysis was performed on existing in vitro experimental results [5] using the 'Fiji' distribution of ImageJ. Two conditions were investigated further for comparison: the wild-type condition and Notch1activated (Tie2-Cre; N1ICD) ventricular cells. These conditions were chosen because the cells migrated mainly in 2D rather than invading the collagen gel, allowing 2D comparisons of cell shape and migration to be made. This allows investigation of the influence of Notch1 protein on cell shape and migration during EMT.

As the wild type condition consisted of a monolayer of endocardial cells, while the Notch1 activated cells were scattered, it was necessary to process them in different ways to extract individual cell shapes. For the wild type condition, first a maximum intensity z-projection of the stack was produced, and the myocardium removed. The blue channel, which represents nuclei of the cells, was overlaid on the image in white. Then Find Maxima in ImageJ was used, which located the local maxima (in terms of brightness) in the image. The output type 'Segmented Particles' was used, which uses a watershed algorithm to extract an outline from the area surrounding the maxima. These outlines were then added to the 'Region of Interest (ROI) Manager', allowing measurement of cell shape metrics to be performed. This workflow is shown in Figure 4.
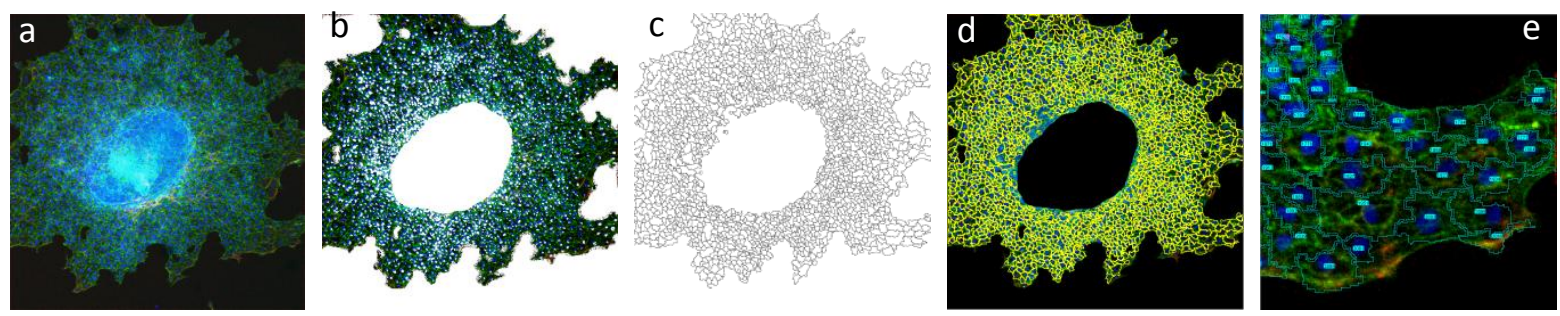

Figure 4 Image processing workflow in ImageJ for wild-type cells. a) Starting image b) Nuclei extracted and overlaid in white, background removed. c) Find maxima used to output 'Segmented Particles' d) Outlines used to add cell shapes to 'ROI Manager' e) Close-up inspection of overlay indicates that cell outlines are a reasonably good fit.

For the Notch1 activated cells, slice number 15 was analysed, as this provided a representative image of cells moving on the surface of the collagen gel. The myocardium from the ventricular explant was removed from the image. 'Adjust Threshold' in ImageJ was used to create a binary image of the cells. 
The 'Analyse Particles' plugin was then used to add the cell shapes to the 'ROI Manager', which was used to measure the cell shape characteristics. This workflow is shown in Figure 5.
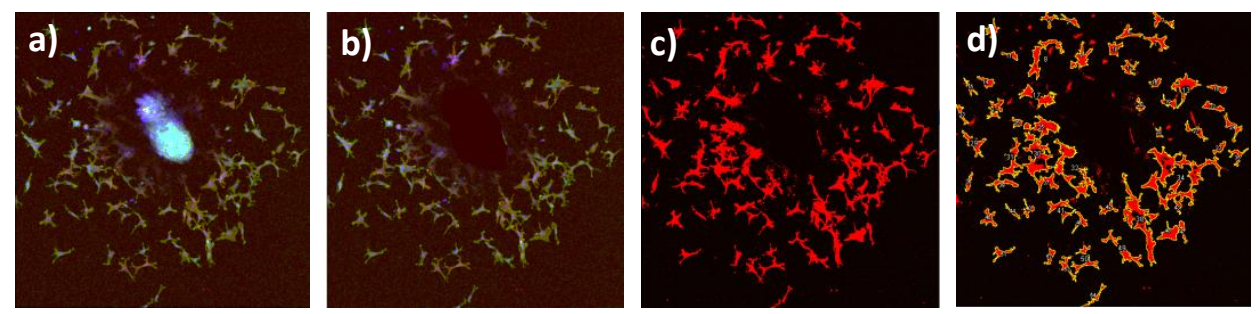

Figure 5 Image processing workflow in ImageJ for Notch1 activated cells undergoing EMT. a) Starting image b) Myocardium removed. c) 'Adjust Threshold' to create binary image d) 'Analyse Particles' used to add cell shapes to 'ROI Manager'.

The use of different methods to extract cell shapes in for the two different cases was necessary, because they had very different characteristics. While this may produce a bias towards different shapes for the different methods, close inspection showed a reasonable match to visible cell outlines. Future work may produce a method that can be applied to both cases.

\subsection{Cell Level Parameters}

In order to account for the effects of Notch signalling on cell adhesion molecules and cytoskeletal proteins, both cell adhesion and cell shape parameters are explored in the simulations. A primary effect of Notch signalling is the disassembly of VE-cadherin adherens junctions, which is accounted for in the models as a loss of cell-cell adhesion. Notch signalling increases the expression of TGF- $\beta$; which in turn increases integrin adhesion molecules. This is accounted for in the model as increased cell-matrix adhesion (reduction in the surface energy parameter between the cell and matrix).

\section{Results}

\subsection{Image Processing Results}

The results of the image processing indicated that there is a significant difference in terms of circularity and aspect ratio between wild-type and Notch1 activated ventricular endocardial cells. As shown in Figure 6, the N1ICD cells have both a significantly less circular morphology, but are not significantly elongated compared to the wild-type endocardial cells.

However, these results do not tell us anything about the mechanism by which Notch signalling induces a shape change in these cells. It is likely to be a combination of many factors. For example, Notch signalling induces a loss of VE-Cadherin protein, which means a loss of endocardial adherens junctions. A secondary effect of cells being able to migrate away from the epithelial layer is that they are no longer pulled into an approximately hexagonal shape by other epithelial cells. Additionally, there is significant crosstalk between the Notch and TGF- $\beta$ signalling pathways [21] and TGF- $\beta 2$ expression is up-regulated in Notch1-activated ventricular endocardial cells [5]. TGF- $\beta$ signalling up-regulates several integrin adhesion molecules, which bind to extracellular matrix components such as collagen, laminin, vimentin and fibronectin [22]. This increased adhesion to the matrix might pull cells into a spindly morphology, as 
well as increasing their migration capacity. Finally, Notch and TGF- $\beta$ induce a relocation of E-cadherin and cortical actin from cell walls to the cytoskeleton, providing the cell with an ability to survive under mechanical stress, and thus change shape [3]. The potential role of these different effects was investigated through simulation models, as reported in the next section.

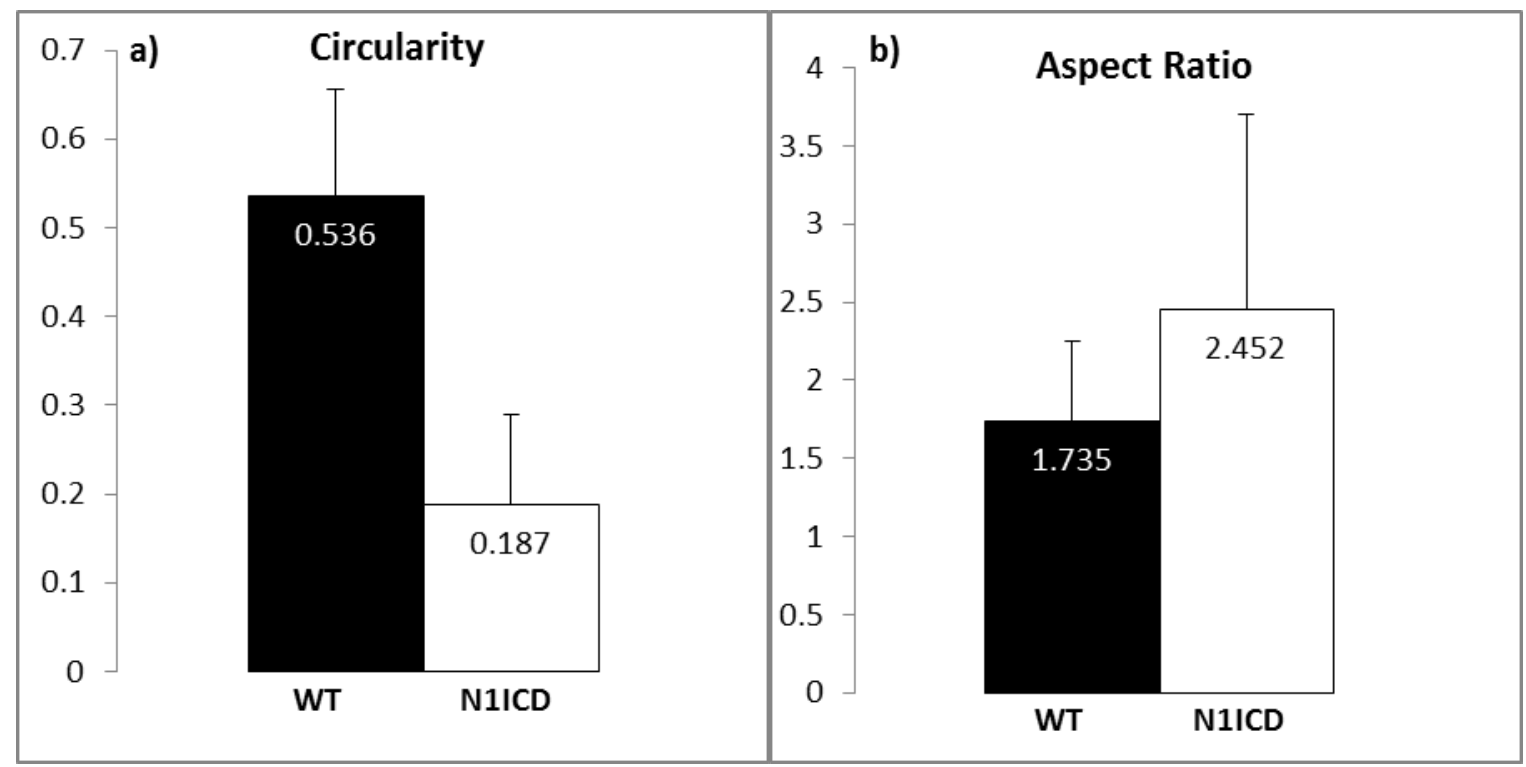

Figure 6 Shape metric comparison of wild-type and N1ICD murine ventricular endocardial cells. a) Wild-type cells have a mean circularity of 0.536 , and N1ICD cells $0.187(p<0.001)$. b) Wild-type cells have a mean aspect ratio of 1.735 and N1ICD cells 2.452, however this difference is not significant. Error bars show standard deviation.

\subsection{Single Cell Simulation Results}

Compucell3D simulations of individual cells were used to simulate changes in cell shape. Starting from a rounded morphology, the effects of an increased surface perimeter (relative to volume), and increased motility were simulated. Cell motility was increased by increasing the $T$ parameter which increases the number of pixel copies accepted, and thus the speed that simulated cells move. Changes in shape led to greater motility, while greater motility led to changes in cell shape; indicating that cell shape and migration are highly interdependent. In each case, the base case of a rounded morphology is used, before changing one parameter at a time. Base case simulations used the parameters:

'lambdaVolume' $=3.0$, 'lambdaSurface' $=3.0$, 'targetVolume' $=225$, 'targetSurface' $=60, T=10$. Contact energy with the surrounding Medium is set to zero. Cells are initialised in a square shape of $15 \times 15$ pixels, which represents a 2D surface area (volume) of $225 \mu \mathrm{m}^{2}$ per cell, assuming a constant thickness of $1 \mu \mathrm{m}$.

For clarity, the parameter values used for all the simulations shown in all the figures in this paper are given in Table 1. 
Table 1 Simulation parameters for Figures 7-13.

\begin{tabular}{|l|l|l|l|l|l|l|l|l|l|l|}
\hline & T parameter & $\begin{array}{l}\text { Target } \\
\text { Volume }\end{array}$ & $\begin{array}{l}\text { Target } \\
\text { Surface } \\
\text { Volume }\end{array}$ & $\begin{array}{l}\text { Lambda } \\
\text { Surface }\end{array}$ & $\begin{array}{l}\text { Lambda } \\
\text { Point } \\
\text { Plasticity } \\
\text { Lambda }\end{array}$ & $\begin{array}{l}\text { Cell-Cell } \\
\text { Contact } \\
\text { Energy }\end{array}$ & $\begin{array}{l}\text { Cell- } \\
\text { Medium } \\
\text { Contact } \\
\text { Energy }\end{array}$ & $\begin{array}{l}\text { Target } \\
\text { Length }\end{array}$ & $\begin{array}{l}\text { Lambda } \\
\text { Length }\end{array}$ \\
\hline Fig. 7 & 10 & 225 & $\begin{array}{l}{[60,90,} \\
120,150]\end{array}$ & 3.0 & 3.0 & - & 0 & 0 & - \\
\hline Fig. 8 & $\begin{array}{l}{[10,50,100,} \\
200,500]\end{array}$ & 225 & 60 & 3.0 & 3.0 & - & 0 & 0 & - \\
\hline Fig. 9 & 20 & 225 & 120 & 3.0 & 3.0 & {$[20,5]$} & {$[-15,0]$} & 0 & - \\
\hline Fig. 10 & 20 & 225 & 60 & 3.0 & 3.0 & {$[0,200]$} & {$[0,-20]$} & 0 & - \\
\hline Fig. 11 & 500 & 225 & 60 & 3.0 & 3.0 & {$[200,0]$} & {$[-20,0]$} & -20 & - \\
\hline Fig. 12 & 500 & 225 & 60 & 3.0 & 3.0 & - & 0 & -200 & - \\
\hline Fig. 13 & 500 & 225 & 60 & 3.0 & 3.0 & - & 0 & -100 & 50 \\
\hline
\end{tabular}

\subsubsection{Larger Surface Area Induces Greater Motility}

An increase in surface area, relative to volume, can be induced by increasing the target surface parameter, while keeping other parameters constant. This constrains cells into adopting a fibroblastic (spindle-shaped) morphology. However this change in morphology is also accompanied by an increase in motility, as shown in Figure 7.
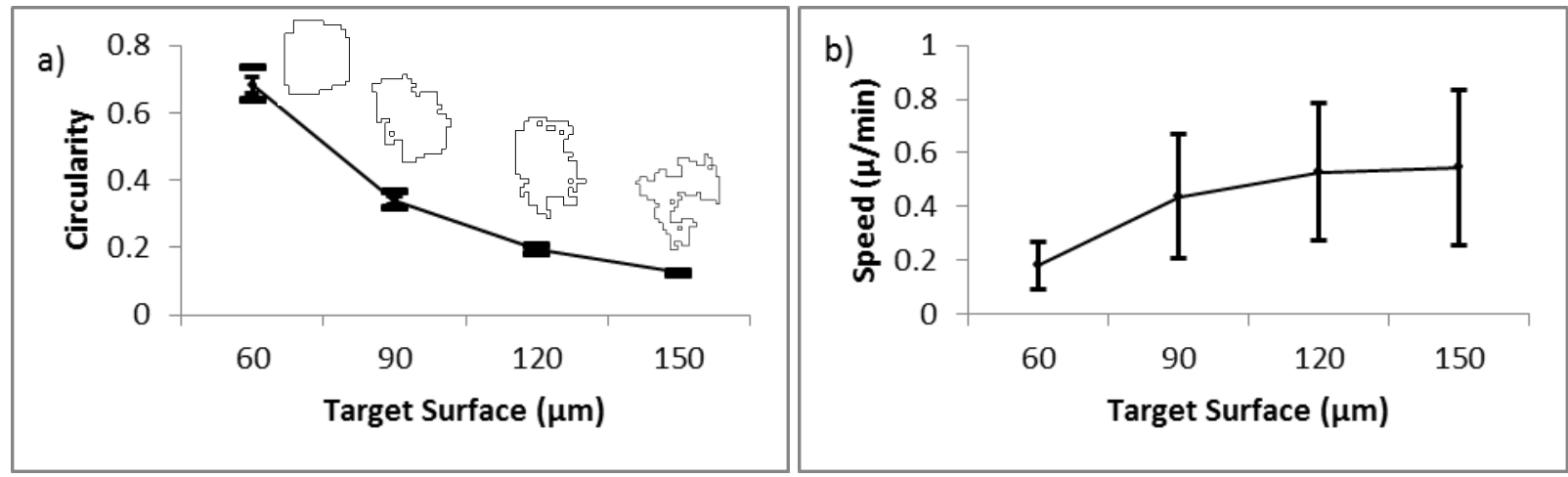

Figure 7 a) Circularity of simulated cells falls with increasing Target Surface. b) This is accompanied by increased speed (and hence motility). Error bars show standard deviation, and caps show the range, from 10 simulation replicas.

This can be explained by the greater number of interactions between cell surfaces and medium leading to a greater number of pixel copies being attempted and accepted. This has the biological equivalent of a cell with a more fibroblastic morphology having a greater surface area over which to interact with and adhere to the matrix.

\subsubsection{Greater Motility Induces Fibroblastic Morphology}


Increasing the value of the $T$ parameter increases the speed of movement of the centre of mass of the cells (as defined in Section 2.4.). However, this increase in cell motility is accompanied by the adoption of a fibroblastic morphology. Furthermore, the magnitudes of reduced circularity associated with increased speeds are similar to those that result from increasing the target surface (compare Figure 7 with Figure 8). In both cases, migration speeds are consistent with those of in vitro epithelial and mesenchymal cells [16].
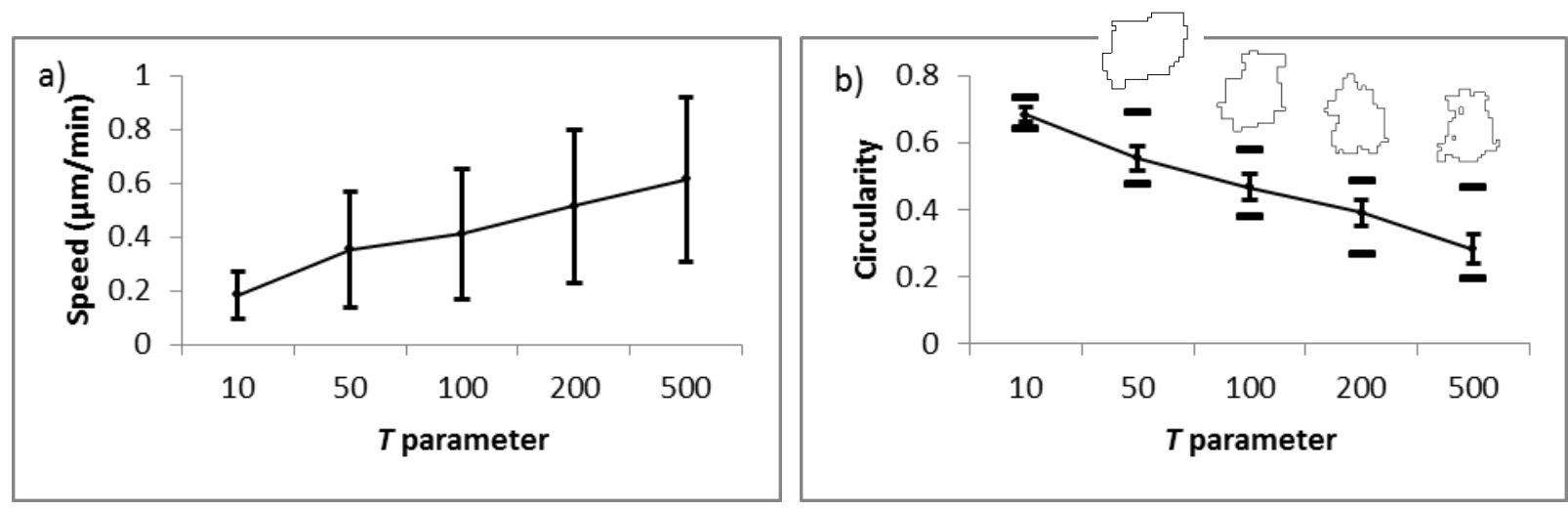

Figure 8 a) Speed of simulated cells increases as a result of increasing the $T$ parameter b) This is accompanied by a reduction in circularity. Error bars show standard deviation, and caps show the range, from 10 simulation replicas.

There are two plausible explanations for this. Either the increase in the $T$ parameter has the side of effect of giving the cells the flexibility to deviate from a rounded morphology; or the easiest (lowest entropy) way for a cell to be more motile is for it to adopt an elongated or fibroblastic morphology. This has the biological equivalent of a cell changing shape in order to permeate the cell matrix.

\subsection{Multi-cell Simulation Results}

Multi-cell simulations were performed which corresponded to the dimensions of the endocardial explants reported in [5]. Each simulation consisted of 316 cells, initially arranged in a filled circle. As with the single cell models, each cell has initial dimensions of $15 \times 15$ pixels $\left(225 \mu \mathrm{m}^{2}\right)$, with target volume parameters that maintain this size. In this multi-cell model four factors are considered that might guide EMT: loss of epithelial labile adhesion; loss of epithelial plastic adhesion; increase in motility; and increase in adhesion to the surrounding matrix. For simplification, an assumption is made that both types of epithelial adhesion would be lost during EMT as a result of down-regulation of VE Cadherin. As discussed in section 1.2, these two phases of adhesion can be modelled as two types of force to investigate their individual roles in driving epithelial morphology. In order to capture the difference in magnitude, labile adhesion is set between 0 and -20 , and the strength of plastic coupling 'lambda' between 0 and 200.

\subsubsection{Labile Adhesion and Junctional Coupling}

Multi-cell simulations incorporate terms for both plastic coupling and junctional adhesion in order to perform a basic parameter scan to investigate the role of these forces together with cell morphology in maintaining epithelial tissue. For endothelial cells, a target surface of $60 \mu \mathrm{m}$ is used, while for mesenchymal cells a target surface of $120 \mu \mathrm{m}$ is used, based on the results of the single cell simulations. 
It was postulated that labile adhesion and junctional coupling might be able to rescue epithelial morphology for cells that were given mesenchymal shape parameters. However, as shown in Figure 9, this was not the case. Cells with adhesion neither maintained contact, nor formed a more rounded morphology. Higher levels of plastic coupling produced incoherent results (not shown).

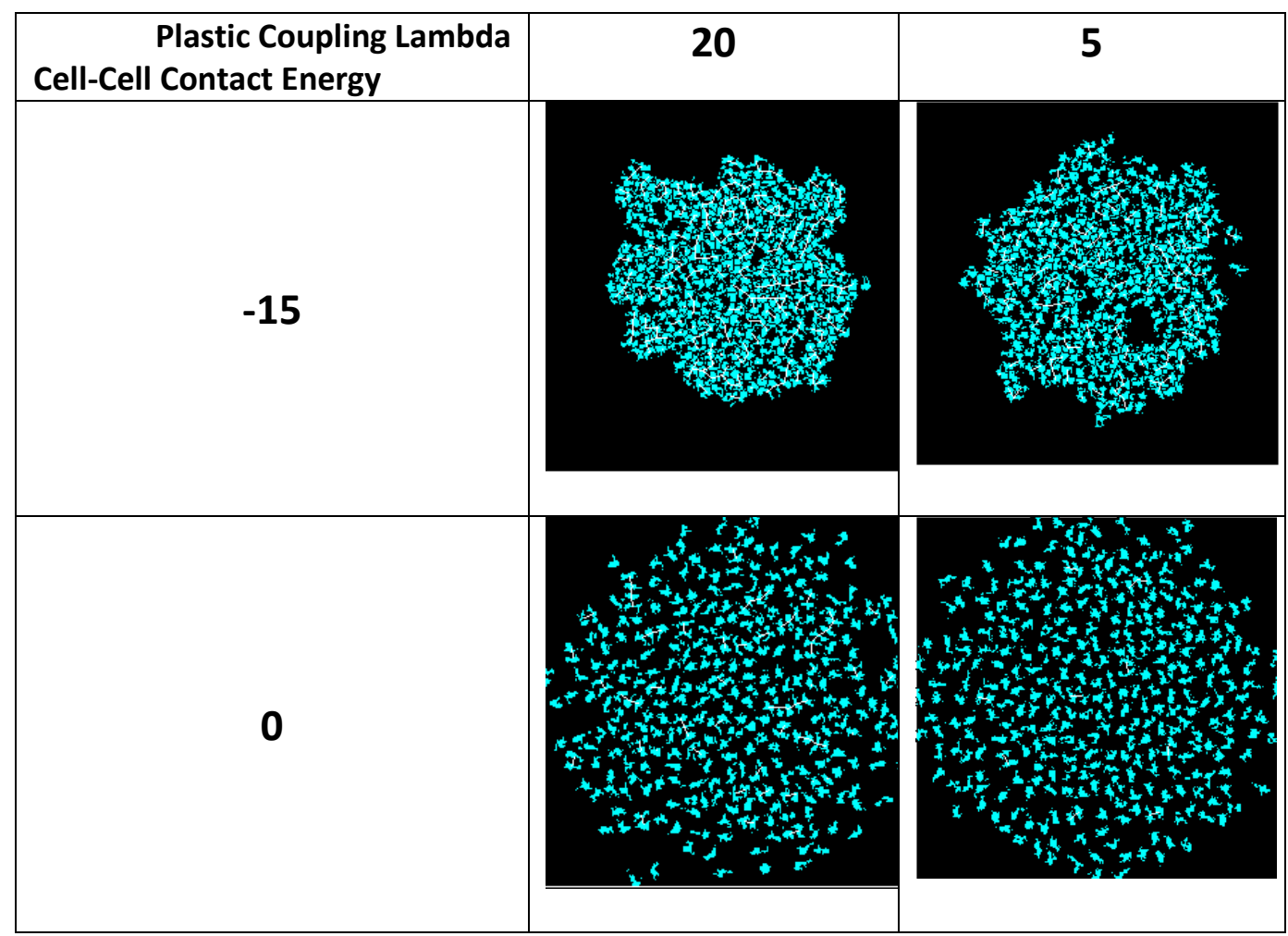

Figure 9 Cells with fibroblastic morphology. Simulation snapshots at $50000 \mathrm{MCS}$, for different levels of plastic coupling lambda $[20,5]$ and cell-cell contact energy $[-15,0]$.

These results suggest that some inherent tendency towards a rounded morphology is required, in addition to the mechanisms of epithelial adhesion. It was also hypothesised that epithelial roundness could be shown to be an emergent property of cellular adhesion, in cells with epithelial shape parameters. However, neither junctional coupling nor labile adhesion significantly increased the roundness of these simulated cells (Figure 10).
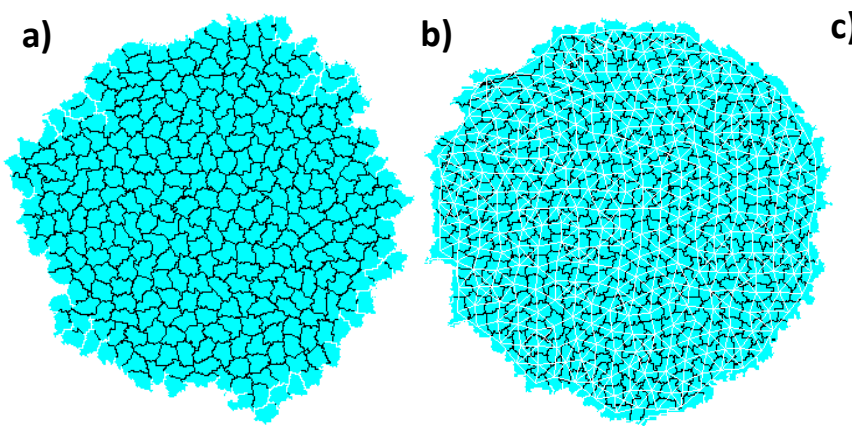

c)

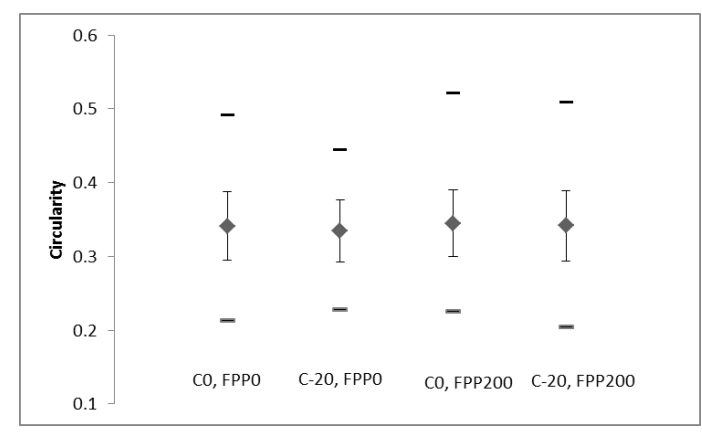


Figure 10 a) Monolayer with no epithelial adhesion and b) monolayer with both labile and junctional adhesion, after 50000 MCS. c) Labile adhesions, C, and junctional coupling, FPP, do not significantly affect average cell roundness ( $p>0.05, n=316$ in all cases).

This suggests that, while a loss of epithelial adhesion may be necessary for cell migration and shape changes observed in EMT, it is not a sufficient condition, as increased motility and/or increased cellmatrix adhesion are also required.

\subsubsection{Increase in Motility}

Based on the results of the single cell simulations, it was postulated that an increase in cellular motility (via the $T$ parameter), coupled with a loss of epithelial adhesion, might be sufficient to induce an EMT process. This was not the case, as the epithelial integrity and morphology were preserved even under large increases in the $T$ parameter value (from 20 to 500). However, when this motility increase is coupled with a moderate increase in cell-matrix adhesion, an intermediate phenotype is observed, whereby a proportion of cells migrate, while maintaining a rounded morphology. The increase in cellmatrix adhesion is modelled by a reduction in cell-matrix contact energy from 0 to -20 . This intermediate phenotype only occurred in the cases with a loss of junctional adhesion, illustrating that junctional adhesion has a greater effect in maintaining epithelial morphology in this case (Figure 11).

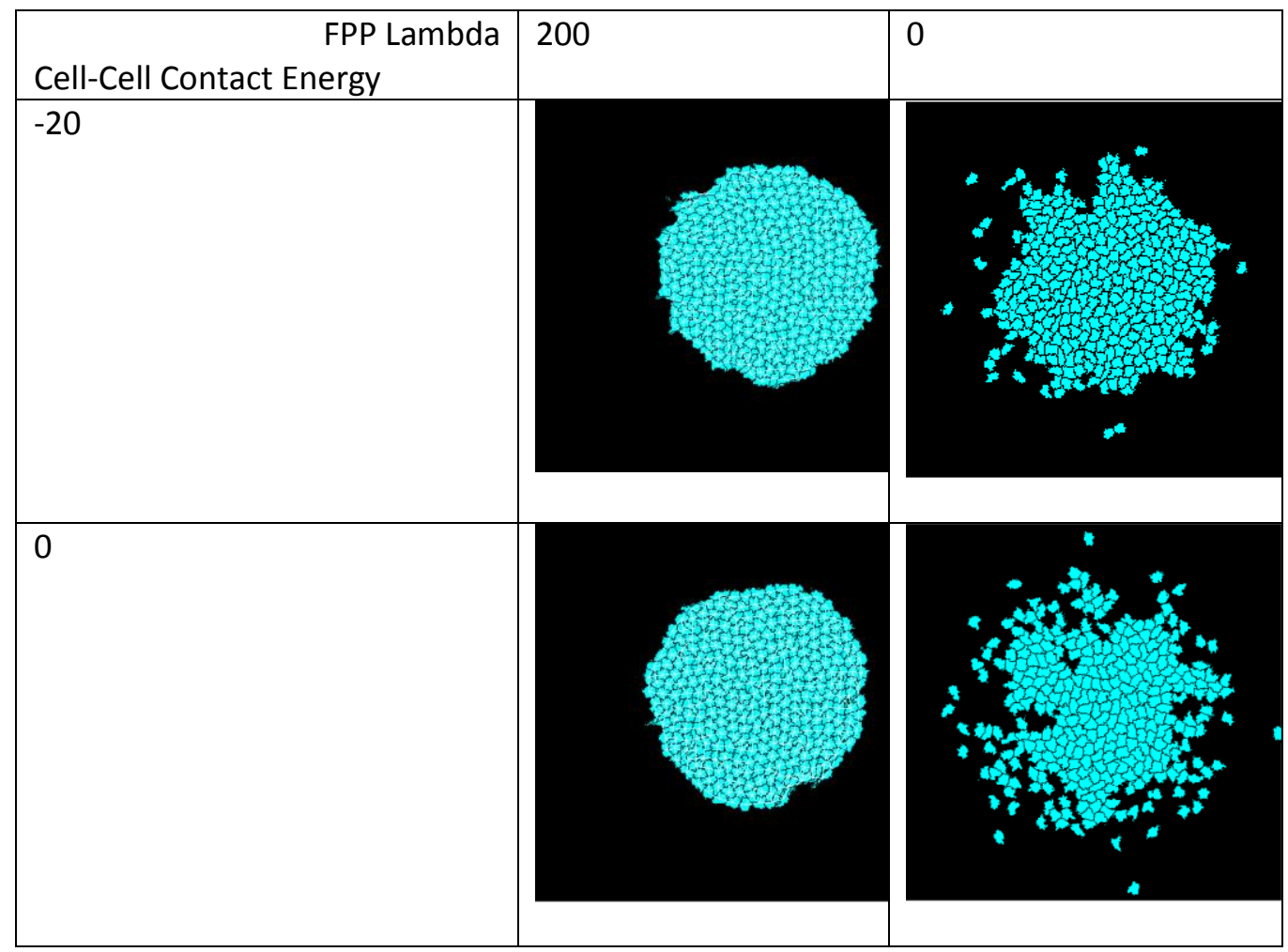

Figure 11 Under conditions of increased motility ( $T=500)$ and moderate cell-matrix adhesion (-20), cells scatter under conditions with a loss of junctional coupling, but not with a loss of labile adhesion alone. In all cases cells maintain a rounded morphology.

The rounded morphology observed in these migratory cells is consistent with an intermediate phenotype observed in endocardial cells for which some Notch target genes (Hey1 or Hey2) have been 
deleted [23]. This suggests that Notch signalling has multiple phenotypic effects in these cells. Loss of cell-cell adhesion is mediated by loss of VE-Cadherin (in endocardial explants), while increase in cellmatrix adhesion is mediated by up-regulation of vimentin and fibronectin. These different effects are induced to a greater or lesser extent by the different Snail genes; which are Notch targets [24]. In the next section the increase in cell-matrix adhesion is investigated.

\subsubsection{Increase in Cell-Matrix Adhesion}

A strong cell-matrix adhesion was added to the model by reducing the cell-matrix contact energy parameter from 0 to -200 . This case, with combined loss of epithelial adhesion, increase in motility and strong increase in cell-matrix adhesion, leads to migration and cell morphology changes consistent with those induced in Notch activated mesenchymal cells (see Figure 12).
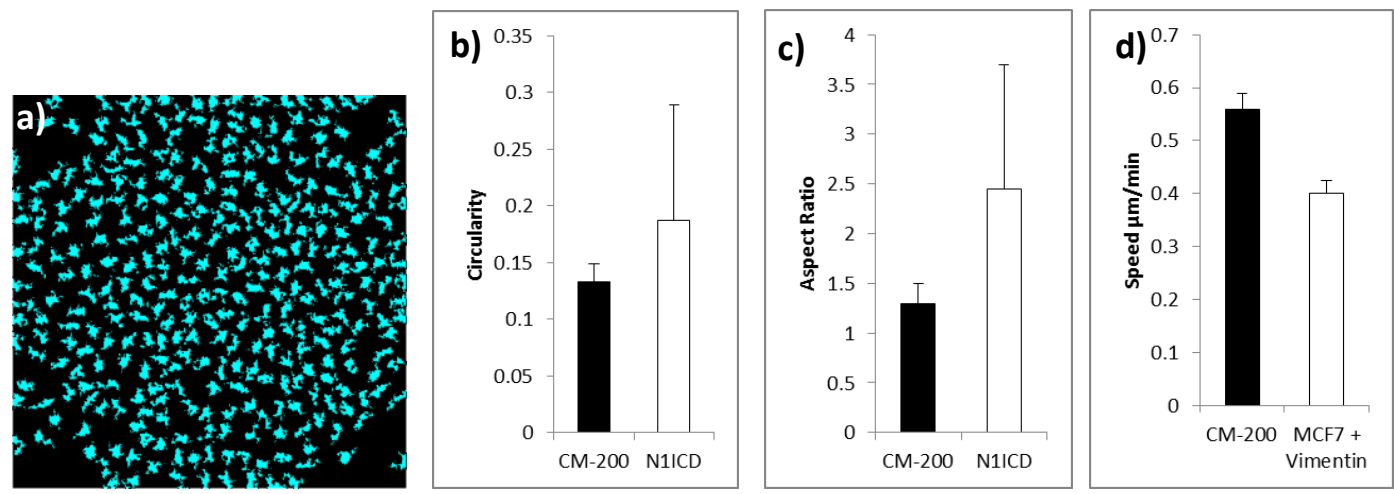

Figure 12 Combined loss of cell adhesion, gain of cell-matrix adhesion and increased motility simulation a) Snapshot at 50000 MCS b,c,d) Mean and standard deviation of simulated (CM-200) cell characteristics at 50000 MCS ( $n=316)$ compared with those obtained from in vitro notch activated endocardial cells (N1ICD, $n=54$ ) [5] or MCF-7 cells transfected with vimentin $(n=5)[16]$.

Simulated and measured values for aspect ratio and circularity were significantly different $(p<0.01)$, indicating that the model does not perfectly represent the morphological characteristics of Notch activated cells. Data are not available to make the comparison for migration speeds. While the values are not a perfect fit, they are consistent with the order of magnitude for the three EMT characteristics. The next section investigates the potential role for cell elongation to improve the model of EMT.

\subsubsection{Cell Elongation}

In order to achieve the large aspect ratio observed in the N1ICD cells, a target length of 50 was set, with a constraint value of 5.0 for all cells in the simulation. Additionally, to increase circularity while reducing speed, the cell-matrix contact energy parameter was reduced from -200 to -100. This produced cell behaviour that was not significantly different from the in vitro N1ICD cells, as shown in Figure 13. 

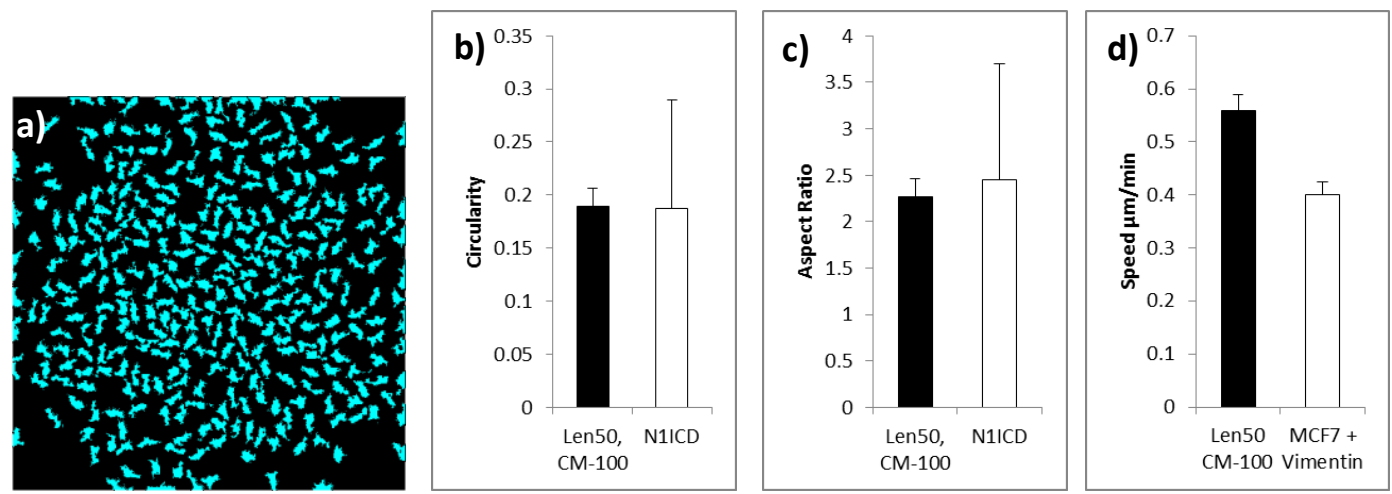

Figure 13 Addition of an elongation parameter to the model, and reduction of cell-matrix adhesion from -200 to -100, provides a better fit, a) Snapshot at 50000 MCS b,c,d) Mean and standard deviation of simulated cell characteristics at 50000 MCS ( $n=316)$ compared with those obtained from in vitro notch activated endocardial cells (N1ICD, $n=54)$ [5] or MCF-7 cells transfected with vimentin $(n=5)[16]$.

Cell speed was not significantly affected by the reduction in cell-matrix adhesion, though it did increase circularity, making the cells morphologically consistent with in vitro N1ICD cells ( $p>0.05)$. The elongation term increased the average aspect ratio of simulated cells, so that they were not significantly different from the in vitro model $(p>0.05)$. As Figure 13 shows the simulation that agrees best with the experimental data, snapshots illustrating the progression from initial state are given in Figure 14.

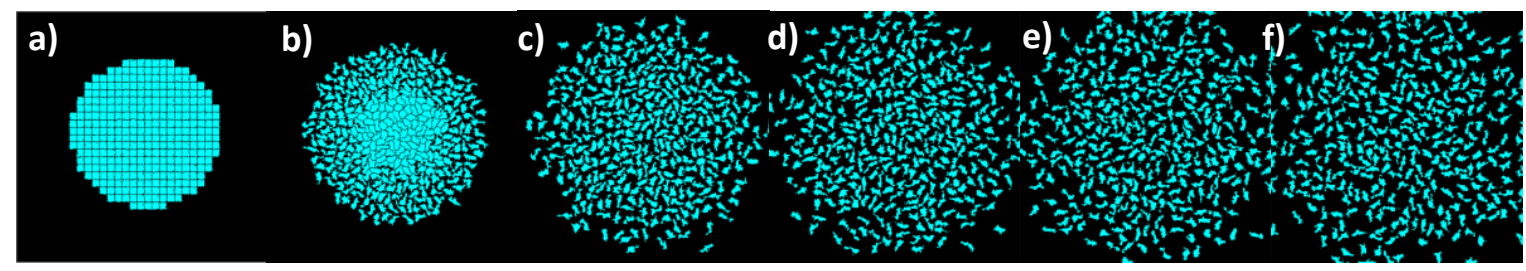

Figure 14 Progression from initial state to final state of simulation giving closest agreement with experimental data a) 0 , b) 1000 , c) 10000 , d) 20000 , e) 30000 , and f) 50000 MCS.

\section{Conclusion}

EMT is a diverse process and is context dependent. EMTs in different contexts are regulated by different signalling pathways and thus different roles may be played by cell morphology, motility and adhesion in each case. The computational model developed in this study provides the flexibility to simulate cells with different combinations of physical parameters. This allows investigation of the conditions under which cells remain epithelial, undergo EMT or exhibit an intermediate phenotype. Cell morphology and migration metrics provide a means to match the model to a particular example of EMT. In combination with in vitro analyses that investigate the effect of individual proteins, this has the potential for quantifying the cell-level effects of gene expression changes. For example, by measuring levels of protein expression that occur when a particular gene is activated, and also measuring changes in cell motility and cell shape, the phenotypic effects of a particular protein can be understood. Modelling and simulation can be used to investigate the physical effects governing a particular phenotype, by fitting a 
model to the measured cell behaviour. In this way, the impact of gene expression on cell level properties could be quantified.

The simulations presented here indicate that Notch1 protein has an effect not only on the loss of cellcell adhesion, but additionally cell motility, cell-matrix adhesion and cell elongation; as these were all necessary to induce migratory and morphological changes in the model that were consistent with those observed in vitro. While the mechanisms of adhesion changes are well known, the model does not suggest a mechanism that may produce increased motility and cell elongation. Additionally, the model developed demonstrated that the intermediate phenotype of rounded migratory cells (observed with deletion Notch target genes Hey1 or Hey2) emerges from loss of epithelial adhesion in the absence of increased epithelial-matrix adhesion. An associated outcome is that cell-matrix adhesion exerts greater influence on cell shape than cell-cell adhesion. In the model, rounded cells have low cell-matrix adhesion, while fibroblastic cells have high cell-matrix adhesion. The model failed to demonstrate control of cell shape by cell-cell adhesion. This is consistent with the intermediate phenotype cells, as they preserve cell roundness (due to low cell-matrix adhesion) despite having impaired cell-cell adhesion (and migrating away from the tissue).

The model has the potential to be applied to more detailed analyses of signalling pathways and the effects of perturbations, by assigning a pathway model to each cell. A Compucell3D function exists for assigning SBML-encoded reaction models to simulated cells, and to pass information between cell and protein level variables. As SBML is a widely used standard interchange format for reaction kinetics modelling, there is the potential for these models to be developed as a separate effort. Multi-scale computational modelling of this form will provide a means to assimilate and represent findings on EMT, as well as other multi-scale biological processes.

\section{References}

[1] P. Savagner, Rise and fall of epithelial phenotype: concepts of epithelial-mesenchymal transition. (Landes Bioscience, Austin TX, 2005).

[2] J. P. Thiery and J. P. Sleeman, Complex networks orchestrate epithelial-mesenchymal transitions, Nature Reviews: Molecular Cell Biology, 7 (2006) 131-142.

[3] J. Zavadil, L. Cermak, N. Soto-Nieves, and E. P. Böttinger, Integration of TGF-beta/Smad and Jagged1/Notch signalling in epithelial-to-mesenchymal transition, The EMBO Journal, 23 (2004) 1155-1165.

[4] W. Song, K. Jackson, and P. G. McGuire, Degradation of type IV collagen by matrix metalloproteinases is an important step in the epithelial-mesenchymal transformation of the endocardial cushions, Developmental Biology, 227 (2000) 606-617. 
[5] L. Luna-Zurita et al., Integration of a Notch-dependent mesenchymal gene program and Bmp2driven cell invasiveness regulates murine cardiac valve formation, The Journal of Clinical Investigation, 120 (2010) 3493-3507.

[6] Y.-S. Chu et al., Force measurements in E-cadherin-mediated cell doublets reveal rapid adhesion strengthened by actin cytoskeleton remodeling through Rac and Cdc42, The Journal of Cell Biology, 167 (2004) 1183-1194.

[7] J. Heuberger and W. Birchmeier, Interplay of cadherin-mediated cell adhesion and canonical Wnt signaling, Cold Spring Harbor Perspectives in Biology, 2 (2010) a002915.

[8] K. Niessen, Y. Fu, L. Chang, P. A. Hoodless, D. McFadden, and A. Karsan, Slug is a direct Notch target required for initiation of cardiac cushion cellularization, The Journal of Cell Biology, 182 (2008) 315-325.

[9] F. A. Meineke, C. S. Potten, and M. Loeffler, Cell migration and organization in the intestinal crypt using a lattice-free model, Cell Proliferation, 34 (2001) 253-266.

[10] A. C. Walter, A Comparison of Continuum and Cell-based Models of Colorectal Cancer, PhD Thesis, The University of Nottingham, UK, 2009.

[11] S. D. Hester, J. M. Belmonte, J. S. Gens, S. G. Clendenon, and J. A. Glazier, A Multi-cell, Multi-scale Model of Vertebrate Segmentation and Somite Formation, PLoS Computational Biology, 7 (2011) e1002155.

[12] T. Sun, S. Adra, R. Smallwood, M. Holcombe, and S. MacNeil, Exploring hypotheses of the actions of TGF-beta1 in epidermal wound healing using a 3D computational multiscale model of the human epidermis, PloS ONE, 4 (2009) e0008515.

[13] I. Ramis-Conde, M. A. J. Chaplain, A. R. A. Anderson, and D. Drasdo, Multi-scale modelling of cancer cell intravasation: the role of cadherins in metastasis, Physical Biology, 6 (2009) 016008.

[14] A. Neagu et al., Computational modeling of epithelial-mesenchymal transformations, Biosystems, 100 (2010) 23-30.

[15] M. H. Swat, S. D. Hester, R. W. Heiland, B. L. Zaitlen, and J. A. Glazier, Multi-Cell Simulations of Development and Disease Using the CompuCell3D Simulation Environment, Methods Mol. Biol., 500 (2009) 361-428.

[16] M. G. Mendez, S. I. Kojima, and R. D. Goldman, Vimentin induces changes in cell shape, motility, and adhesion during the epithelial to mesenchymal transition, FASEB Journal, 24 (2010) 18381851.

[17] C. Gilles et al., Vimentin contributes to human mammary epithelial cell migration, Journal of Cell Science, 112 (1999) 4615-4625. 
[18] P. Rupp, R. Visconti, A. Czirók, D. A. Cheresh, and C. D. Little, Matrix Metalloproteinase 2-Integrin av $\beta 3$ Binding Is Required for Mesenchymal Cell Invasive Activity but Not Epithelial Locomotion: A Computational Time-Lapse Study, Molecular Biology of the Cell, 19 (2008) 5529 -5540.

[19] J. de Rooij, A. Kerstens, G. Danuser, M. A. Schwartz, and C. M. Waterman-Storer, Integrindependent actomyosin contraction regulates epithelial cell scattering, The Journal of Cell Biology, 171 (2005) 153-164.

[20] L. McKeen-Polizzotti, K. M. Henderson, B. Oztan, C. C. Bilgin, B. Yener, and G. E. Plopper, Quantitative metric profiles capture three-dimensional temporospatial architecture to discriminate cellular functional states, BMC Medical Imaging, 11 (2011) 11.

[21] Y. Fu et al., Differential regulation of transforming growth factor beta signaling pathways by Notch in human endothelial cells, The Journal of Biological Chemistry, 284 (2009) 19452-19462.

[22] C. Margadant and A. Sonnenberg, Integrin-TGF-beta crosstalk in fibrosis, cancer and wound healing, EMBO Reports, 11 (2010) 97-105.

[23] A. Fischer et al., Combined loss of Hey1 and HeyL causes congenital heart defects because of impaired epithelial to mesenchymal transition, Circulation Research, 100 (2007) 856-863.

[24] A. Barrallo-Gimeno and M. A. Nieto, The Snail genes as inducers of cell movement and survival: implications in development and cancer, Development (Cambridge, England), 132 (2005) 31513161. 